\title{
Assessment of Spatial Distribution Uniformity in 2-Dimensional Discrete Space
}

\author{
Liang Qiu and Dong C. Liu \\ Sichuan University, School of Computer Science, Sichuan China, 610065 \\ Trickster9@163.com
}

\begin{abstract}
This paper proposes an algorithm to assess the spatial distribution uniformity in 2-dimensional discrete space. Spatial distribution uniformity refers to how uniform some objects distribute in a space. The algorithm takes a matrix of logical values that represents the distribution pattern as the input and outputs a number that represents the degree of the uniformity. A pseudo energy attenuation model and the uniformity coefficients in the irrigation field are leveraged in this algorithm. Experiment results show that this algorithm is effective in assessing spatial distribution uniformity in 2-dimensional discrete space.
\end{abstract}

Keywords: Distribution Uniformity; Spatial; Uniformity Coefficient; 2-Dimensional

\section{Introduction}

Distribution uniformity refers to how uniform some objects or quantities distribute in some space. Originally, it was proposed in irrigation field. The distribution uniformity of watering quantities of samples is formulated by uniformity coefficients. Although uniformity coefficients have good performance of the assessment of quantitative distribution uniformity, they show no spatial distribution features of the samples. In the texture analysis field, the distribution uniformity of gray level of a digital image is formulated by the angular second moment (energy) of gray level co-occurrence matrix (GLCM for short) [1], which represents not only the quantitative distribution uniformity of gray levels, but also spatial relationships between pixels. However, the performance of the assessment of spatial distribution uniformity by GLCM is poor. Based on this fact, an algorithm is proposed in this paper to assess the spatial distribution uniformity in 2-dimensional discrete space effectively by combining a pseudo energy attenuation model with the uniformity coefficients. Moreover, how the algorithm evolves from an initial plan to a maturing solution is also presented with the experiment results following.

\section{Related Work}

\subsection{Energy of GLCM}

The gray level co-occurrence matrix (or gray tone spatial-dependence matrix) is a matrix that is defined over an image to be distribution co-occurring values at a given offset. Mathematically, a gray level co-occurrence matrix $C$ is defined over an $n \times m$ image $I$, parameterized by an offset $(\Delta x, \Delta y)$, as:

$$
C_{\Delta x, \Delta y}(i, j)=\sum_{p=1}^{n} \sum_{q=1}^{m}\left\{\begin{array}{l}
1, \text { if } I(p, q)=i \text { and } I(p+\Delta x, q+\Delta y)=j \\
0, \text { otherwise }
\end{array}\right.
$$

where $i$ and $j$ are the gray level values of the image, $p$ and $q$ are the spatial position in the image $I$ and the offset $(\Delta x, \Delta y)$ defines how the pixels are paired up.

The angular second moment (ASM for short), also refers to the energy, of the GLCM is a measure of homogeneity of the image, which is formulated as: 


$$
A S M=\sum_{i=0}^{L} \sum_{j=0}^{L} C(i, j)^{2}
$$

where $L$ represents the maximum of the gray level value [2].

The ASM of GLCM is a good measure of the thickness and sharpness of the texture. Sometimes thin texture may indicate good distribution uniformity of gray level while thick texture may indicate bad distribution uniformity of gray level. However, it is not an efficient assessment of distribution uniformity since the thickness and sharpness of texture is not fully equivalent to the distribution uniformity of gray level and the value of ASM is parameterized by the offset.

\subsection{Uniformity Coefficients}

In irrigation field, various uniformity coefficients have been developed to measure the distribution uniformity of the watering quantity of a number of samples.

The most popular and simplest uniformity coefficient is the low quarter coefficient [3] that is calculated with the equation:

$$
L C=\frac{\overline{D_{l q}}}{\overline{\overline{D a l l}_{\text {all }}}}
$$

where $\overline{D_{l q}}$ represents the average of the lowest quarter of samples and $\overline{D_{\text {all }}}$ represents the average of all samples.

However, Christianse' uniformity coefficient [4] was first used to introduce a uniformity coefficient to the irrigation system [5] which is formulated as:

$$
C U=\left(1-\frac{\left.\sum_{i=1}^{m}\left|x_{i}-u\right|\right]}{\sum_{i=1}^{n} x_{i}}\right) \times 100 \%
$$

where $x_{i}$ is the sample value each and $u$ is the mean of all samples.

Wilcox and Swales used the same method used by Christianse, except that they used squares of the deviation from the mean instead of the deviation themselves [6]. They proposed their equation as:

$$
W U=\left(1-\frac{\sigma}{u}\right) \times 100 \%
$$

where $\sigma$ and $u$ are the standard deviation and mean of the samples respectively.

Benami and Hore proposed their uniformity coefficient [7] that is calculated from the formula:

$$
B U=\frac{N_{h}\left(2 s_{1}+D_{l} M_{L}\right)}{N_{l}\left(2 s_{h}+D_{h} M_{h}\right)}
$$

where:

$N_{h}$ - number of elements greater than the overall mean

$N_{l}$ - number of elements smaller than the overall mean

$M_{h}$ - mean of the elements greater than the overall mean

$M_{l}$ - mean of the elements smaller than the overall mean

$S_{h}$ - sum of the elements greater than $M_{h}$

$S_{l}$ - sum of the elements smaller than $M_{l}$

$D_{h}$ - difference between the numbers of elements smaller and greater than $M_{h}$

$D_{l}$ - difference between the numbers of elements smaller and greater than $M_{l}$

The low quarter coefficient is for stressing the minimum of sample values which makes it only applicable to sprinkler system [8]. It does not work well as a general solution to assessment of uniformity.

Zoldoske proposed that Christianse's coefficient does not emphasize the influence of the sample that is significantly different from the mean on the coefficient since the nonuniformity has a linear relationship with the deviation [9]. Wilcox's coefficient is better than Christianse's coefficient in this aspect.

Although Benami's coefficient was claimed to make the differences between distribution patterns more pronounced, it may produce result that above $100 \%$ in some cases [10]. 
Korven has evaluated these three uniformity coefficients based on correlation and linear regression analysis and proposed that there is little difference between them [11]. However, Korven suggested that Wilcox's uniformity coefficient is the most practical coefficient to use because it is sounder statistically than the other two and it is easier to calculate than Benami's coefficient.

\section{Assessment Algorithm}

\subsection{Preliminary}

The algorithm takes a 2-dimensional matrix of logical values as the input, which illustrates the distribution pattern. The objects of interest are considered as particles that have no size or shape. The value of each element of the matrix represents whether there exists an object. The position of an element is defined as the row-column index pair in the matrix. The distance between two elements is the Euclidean distance between the positions of them. An element with value True is called a full element that indicates that there exists an object and that with value False is called an empty element that indicates no object. Each full element is considered as a point energy source. The energy from a full element attenuates as the distance from the source increases and absorbed by all elements. The weight of an element is the sum of energy it absorbs from all sources. A statistic calculated on the weights is the output, which is the degree of the spatial uniformity of the distribution pattern.

For a distribution pattern of good spatial distribution uniformity, the full elements are all over the distribution pattern matrix which makes the weight of each element is similar to each other. Therefore, the distribution of the weights has good quantitative uniformity. However, for a distribution pattern of bad spatial distribution uniformity, the full elements gather into one or several clusters, which make the significant differences among the weights. Therefore, the distribution of the weights has bad quantitative uniformity. This algorithm makes the statistic that assesses the quantitative distribution uniformity of the weights represent the spatial distribution uniformity of the full elements, i.e., the objects of interest.

The following subsections illustrate the key issues of this algorithm, along with some samples of the experiment results. Figure 1 illustrates some samples of the inputs of the experiments, which are some typical distribution patterns. The distribution patterns are illustrated by monochrome images in the size of $200 \times 150$ that the white pixels represent full elements and the black pixels represent empty elements. It is expected that distribution pattern 1 and distribution pattern 2 have the same result of best uniformity and the ranks of the results of the rest distribution patterns are DP4 $>$ DP5 $>$ DP9 $>$ DP7 $>$ DP8 $>$ DP6 $>$ DP3 (DP is the abbreviation of distribution pattern). 

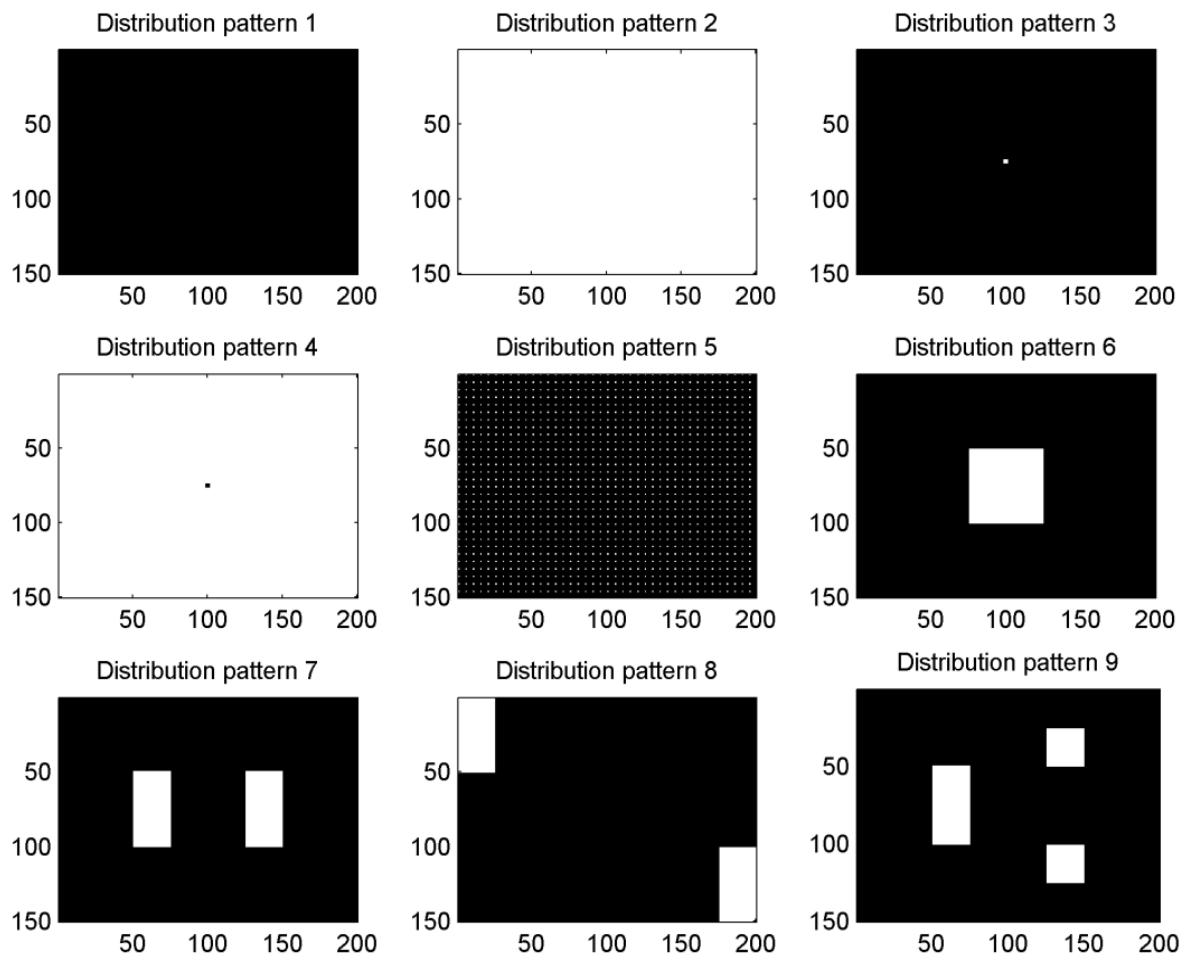

\section{Figure 1. Distribution Patterns}

\subsection{Energy Attenuation Function}

The energy attenuation function should be always positive and monotonically decreasing in $(0,+\infty)$. It should approach to zero at $+\infty$. The differential of the weights of 2 neighboring elements should be consistent with the distribution pattern, which satisfy a criterion that is described as:

Criterion 1 For a full element A and an empty element B that are neighboring, the weight of $\mathrm{A}$ is greater than the weight of $\mathrm{B}$.

Therefore, the second derivation of the energy attenuation function should be positive in $(0,+\infty)$.

Many energy attenuation models use Beer-Lambert law [12] that is formulated as:

$$
I=I_{0} e^{-\alpha x}
$$

where $I$ is the measured energy intensity, $I_{0}$ is the incident energy intensity, $x$ is the transmission distance and $\alpha$ is the attenuation coefficient that is always positive. However, a big attenuation coefficient makes little effect on far away positions. A small one gives too much weight to the neighbors of the source to manifest the distribution. Therefore, the coefficient $\alpha$ is parameterized by the dimension of the distribution pattern, which complicates the definition of the attenuation function.

The energy attenuation function in this algorithm does not have to fully subject to physical facts. Therefore, the reciprocal function $f(x)=1 / x$ is chosen to be the energy attenuation function. However, the reciprocal function value of 0 is infinity. To meet the criteria previously described, the energy attenuation function value of 0 is defined as 3 . Hence, the sources are well stressed and the energy attenuation function is not parameterized by the dimension of the distribution pattern. 


\subsection{Energy Outside of Space of Interest}

The energy of a full element spreads omnidirectional to infinity. However, only the part that is near the source in some extent is interested. For a distribution pattern in the size of $m \times n$, the energy transmission extent is considered as $(2 m-1) \times(2 n-1)$, which is adequate to make the source effect on all elements. However, there is always part of the energy transmission extent outside of the space of interest. Ignoring it makes the energy transmissions of full elements different from each other, which leads to irrational weight distribution.

To make the whole energy transmission extent be applied, the distribution pattern is extended. For a distribution pattern in the size of $m \times n$, it is extended in the size of $\left(m+2 \sqrt{m^{2}+n^{2}}\right) \times\left(n+2 \sqrt{m^{2}+n^{2}}\right)$. The original distribution is placed in the center of the extended pattern and other elements are set as empty elements. The weight calculation and statistic calculation are based on the extended distribution pattern. Figure 2 illustrates the mesh graphs of the weight distributions of the extended distribution patterns illustrated in Figure 1. Most distribution patterns can be obviously inferred from the corresponding mesh graphs of weight distributions. However, some distribution patterns have the mesh graphs of weight distributions that indicate the original distribution patterns obscurely. For example, although the mesh graph of weight distribution of distribution pattern 2 is expected to be a plane, it is actually a big hill.
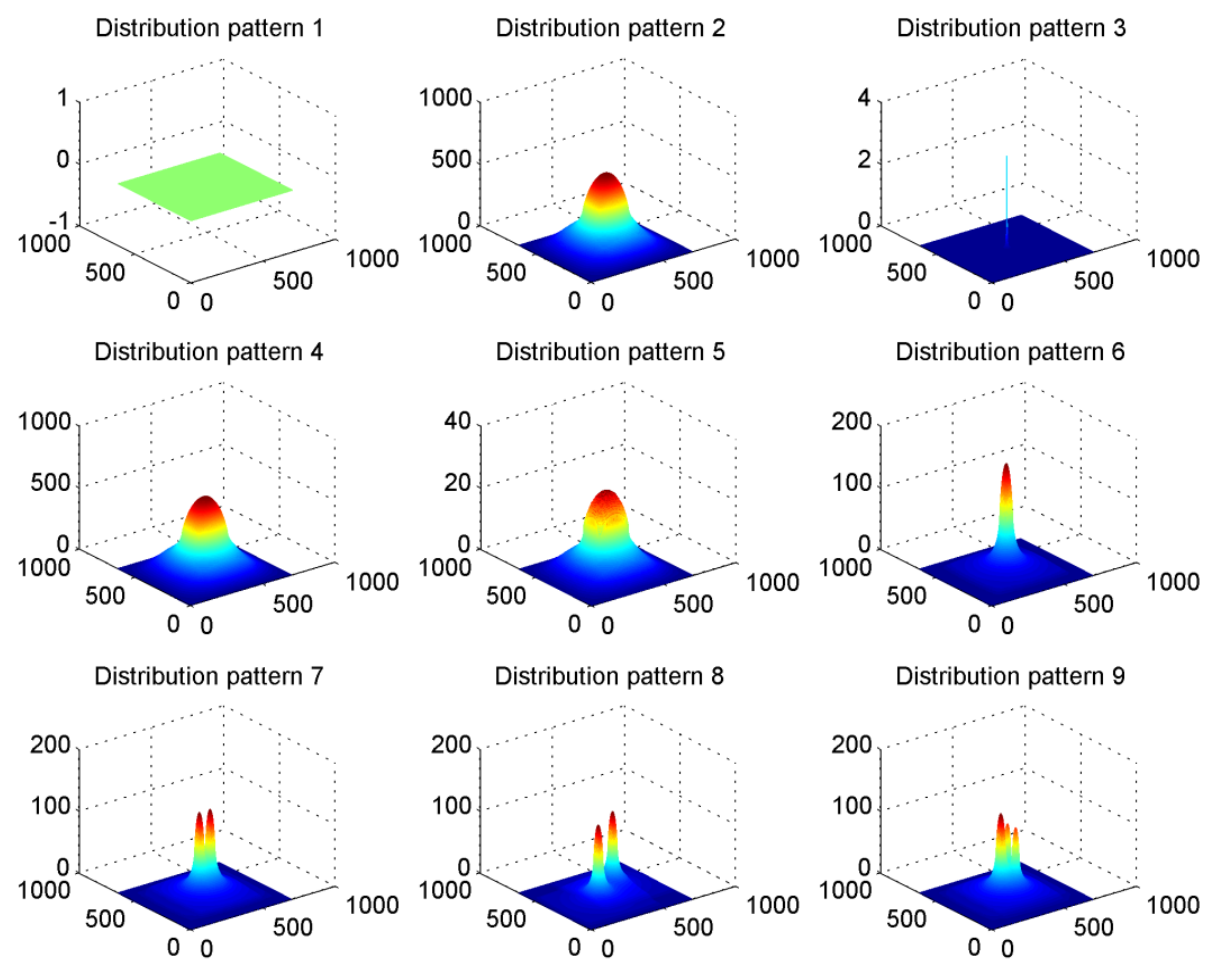

\section{Figure 2. Mesh Graphs of Weight Distributions of Extended Distribution Patterns}

Another solution to this issue is to cyclize the distribution pattern. The opposite edges are rounded up when calculating the weights. For a full element at $\left(x_{0}, y_{0}\right)$ in a distribution pattern in the size of $m \times n$, its energy at the transmission offset of $(\Delta x, \Delta y)$ effects on the weight at $\left(x_{w o} y_{w}\right)$, which is defined as: 


$$
x_{w}=\left\{\begin{array}{l}
x_{0}+\Delta x, \text { if } 0 \leq x_{0}+\Delta x \leq m-1 \\
x_{0}+\Delta x-m, \text { if } x_{0}+\Delta x>m-1 \\
x_{0}+\Delta x+m, \text { if } x_{0}+\Delta x<0
\end{array} \text { and } y_{w}=\left\{\begin{array}{l}
y_{0}+\Delta y, \text { if } 0 \leq y_{0}+\Delta y \leq m-1 \\
y_{0}+\Delta y-m, \text { if } y_{0}+\Delta y>m-1 \\
y_{0}+\Delta y+m, \text { if } y_{0}+\Delta y<0
\end{array}\right.\right.
$$

Figure 3 illustrates the mesh graphs of the weight distributions of the cyclized distribution patterns illustrated in Figure 1, which indicate the distribution patterns more pronouncedly.
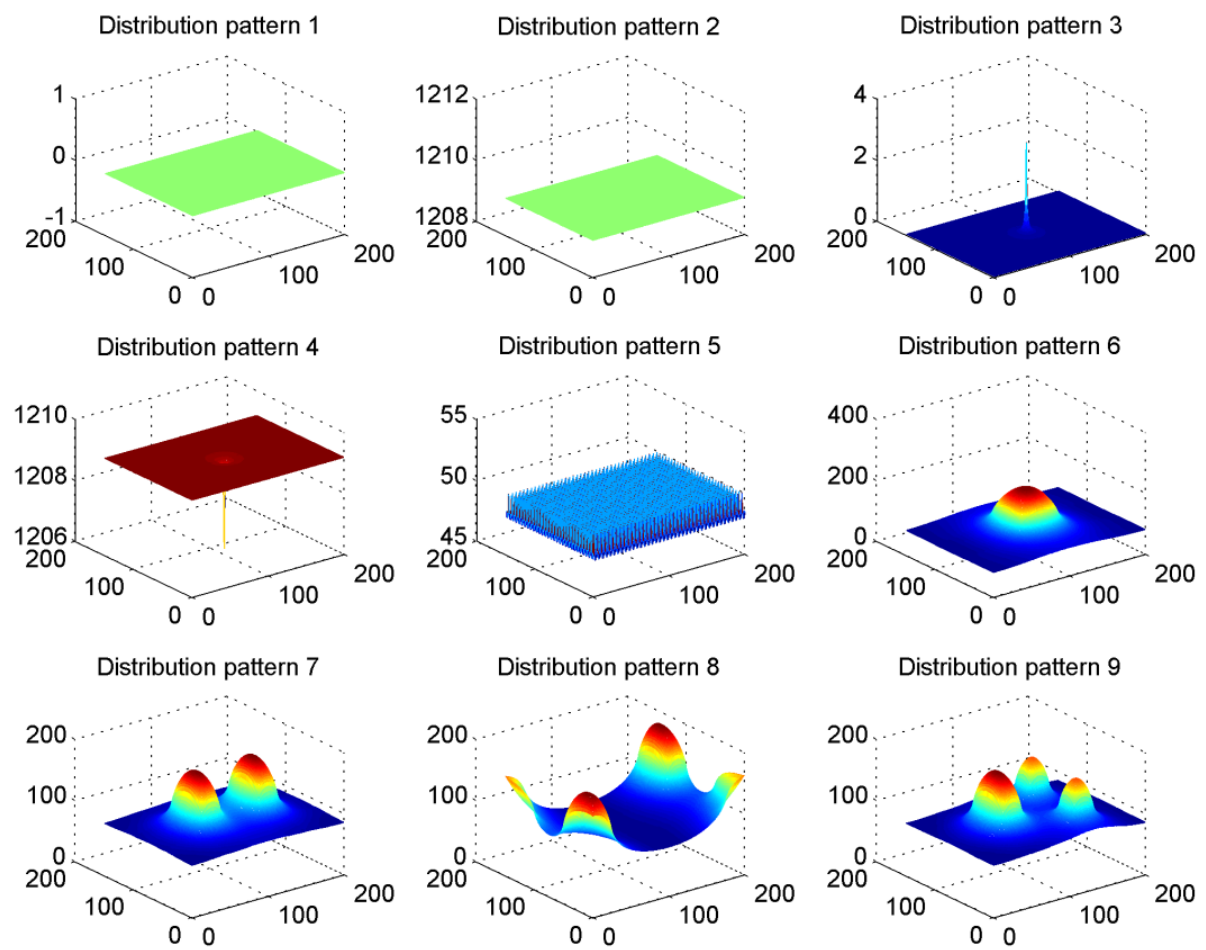

\section{Figure 3. Mesh Graphs of Weight Distributions of Cyclized Distribution Patterns}

\subsection{Quantification Statistic}

A statistic is used to assess the quantitative distribution uniformity of the weights of the distribution pattern, which is the output of this algorithm. The uniformity coefficients are the candidates for the statistic. However, the low quarter coefficient indicates little fluctuation of the weights such that it is not an appropriate candidate for the statistic. In the extreme case that all weights are zeros, there is no arithmetical meaning for Christianse's coefficient and Wilcox's coefficient since they make zero the denominator. Therefore, the value of Christianse's coefficient and that of Wilcox's coefficient in this case are defined as 1 . Moreover, in the cases that the weights are all the same, Benami's coefficient is zero, which indicates the best distribution uniformity instead of worst distribution uniformity. Figure 4 illustrates the uniformity coefficients of the weight distributions illustrated in Figure 2 and Figure 5 illustrates the uniformity coefficients of the weight distributions illustrated in Figure 3. 


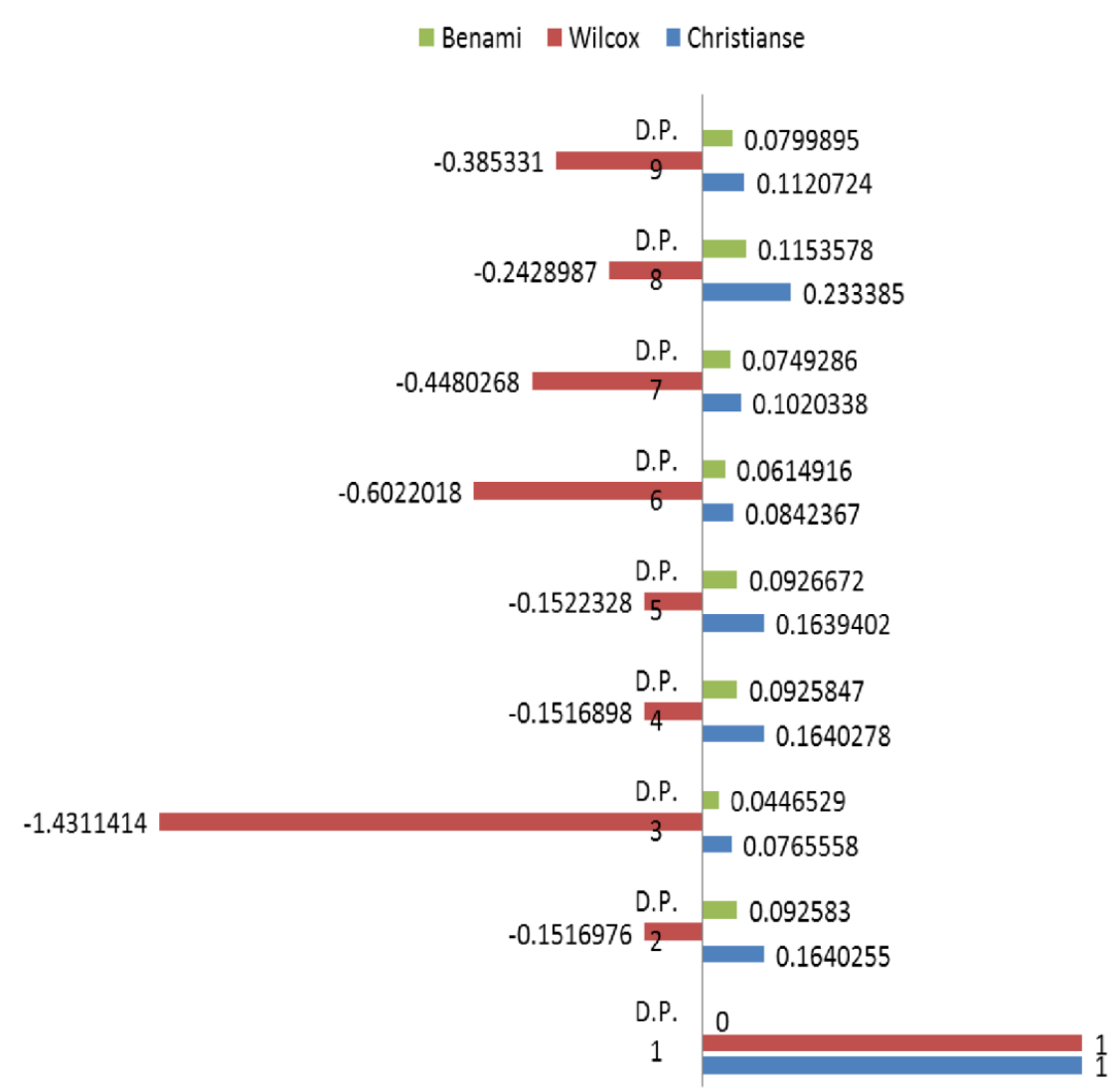

Figure 4. Uniformity Coefficients of Weight Distributions of Extended Distribution Patterns

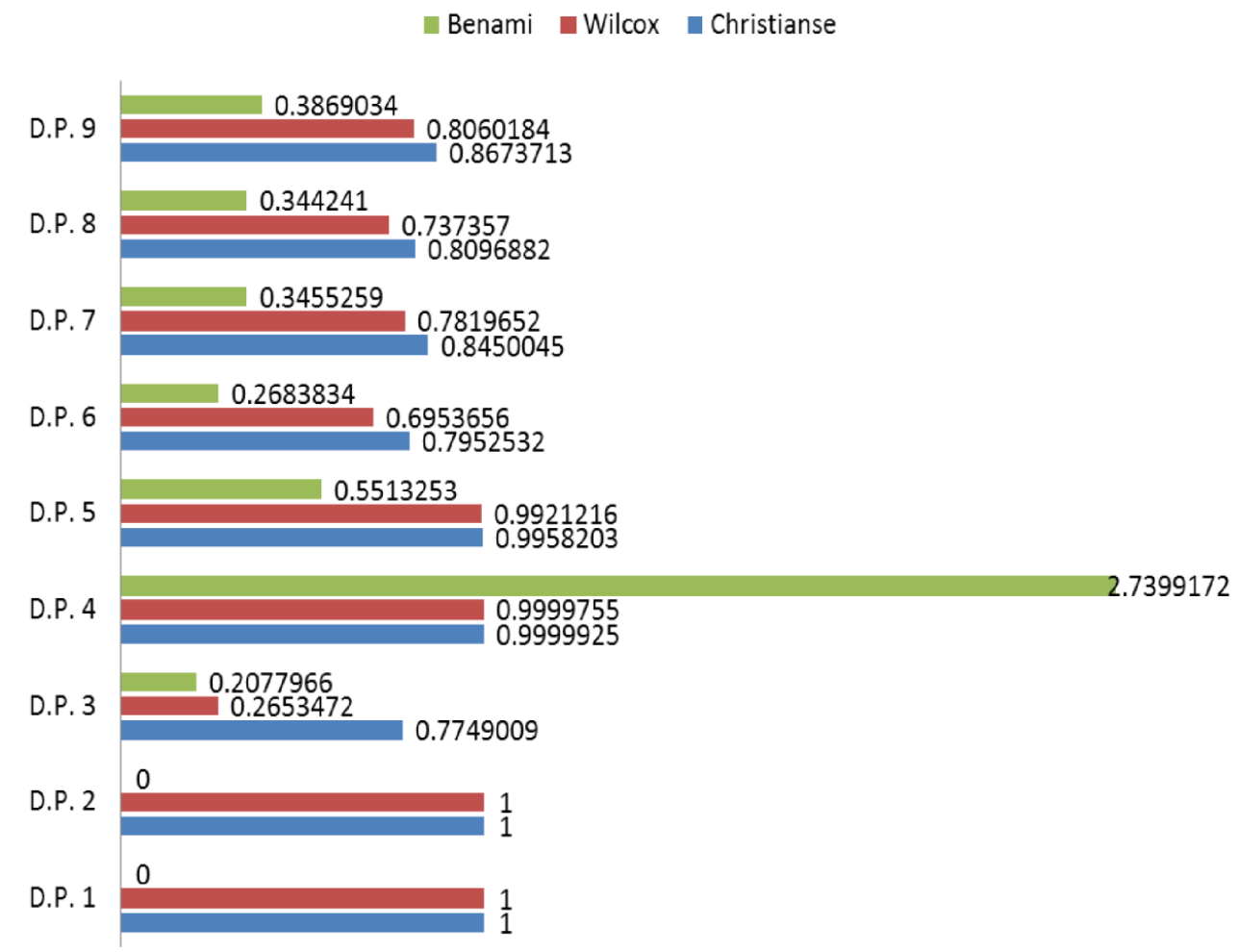

Figure 5. Uniformity Coefficients of Weight Distributions of Cyclized Distribution Patterns 


\section{Conclusion}

The results of extended distribution patterns do not fully match the rank expectation while that of cyclized distribution patterns match the rank expectation perfectly. Therefore, the distribution patterns should be cyclized in this algorithm. The three uniformity coefficients have the same ranks of results in cyclized distribution patterns. However, they have different ranks of results in extended distribution patterns. The performance of Christianse's coefficient is low for the cases of extremely bad uniformity. The range of Benami's coefficient is hard to define and its value does not increase exactly as uniformity becomes better since zero represents the best uniformity. Therefore, Wilcox's coefficient is the most effective one to be used as the quantification statistic.

Experiment results indicate that this algorithm is effective to assess spatial distribution uniformity in 2-D discrete space. The output of this algorithm can be used to represent the degree of the spatial uniformity of a distribution pattern. However, only the spatial distribution uniformities of distribution patterns that have the same size of spaces of interest are comparable by this algorithm.

\section{Acknowledgements}

This work was supported in part by the Foundation of China and Natural Science Foundation of Sichuan Province (Grant No. 2013GZX0147-3) and Crossing Research Project (13H0292).

\section{References}

[1] L. Ma and Y. Fan, “Analysis of texture images”, Science Press, (2009). (in Chinese)

[2] R. M. Haralick, K. Shanmugam and I. H. Dinstein, "Textural features for image classification", Systems, Man and Cybernetics, IEEE Transactions on, vol. 6, (1973), pp. 610-621.

[3] J. L. Merriam and J. Keller, "Farm irrigation system evaluation: A guide for management", Farm irrigation system evaluation: a guide for management, (1978).

[4] J. E. Christiansen, "Irrigation by sprinkling", University of California, (1948).

[5] D. Karmeli, "Estimating sprinkler distribution patterns using linear regression", Transactions of the ASAE American Society of Agricultural Engineers, vol. 21, (1978).

[6] J. C. Wilcox and G. E. Swailes, "Uniformity of water distribution by some undertree orchard sprinklers", Scientific Agriculture, vol. 27.11, (1947), pp. 565-583.

[7] A. Benami and F. R. Hore, "A new irrigation-sprinkler distribution coefficient", Transactions of the ASAE, vol. 7.2, (1964), pp. 157-158.

[8] W. Han, P. Wu, Q. Yang and H. Feng, "Advances and Comparisons of uniformity evaluation index of sprinkle irrigation", Transactions of the CSAE, vol. 21.9, (2006), pp. 172-177. (in Chinese)

[9] D. F. Zoldoske, K. H. Solomon and E. M. Norum, "Uniformity measurements for turfgrass: What's best", Irrigation Notes: Center for Irrigation Technology, Fresno, CA, (1994).

[10] E. Maroufpoor, A. Faryabi, H. Ghamarnia and G. Y. Moshrefi, "Evaluation of uniformity coefficients for sprinkler irrigation systems under different field conditions in Kurdistan Province (northwest of Iran)", Soil and Water Research, vol. 5.4, (2010), pp. 139-145.

[11] H. C. Korven, "An Evaluation of Three Coefficients as a Measure of Uniformity of Water Application by Sprinklers", CANADIAN AGRICULTURAL ENGINEERING, vol. 10.2, (1968).

[12] A. Beer, "Bestimmung der Absorption des rothen Lichts in farbigen Flüssigkeiten", Annalen der Physik, vol. $162.5,(\mathbf{1 8 5 2})$, pp. $78-88$. 